\title{
Outcomes after posterior fixation of iris-claw lens in aphakic eyes
}

\author{
Sangeetha ${ }^{1, *}$, Kanthamani' ${ }^{2}$, Borra Harish Laxman ${ }^{3}$, Chirra Reddy Gari Meghana ${ }^{4}$ \\ ${ }^{\mathbf{1}}$ Assistant Professor, ${ }^{2}$ Professor, ${ }^{3,4}$ Junior Resident, Sri Devaraj Urs Medical College, Kolar, Karnataka, India
}

*Corresponding Author: Sangeetha

Email: sangeetha31jayakumar@gmail.com

\begin{abstract}
Purpose: To analyze and determine the postoperative visual acuity and complications after intraocular implantation of an iris-claw lens in aphakic patients.

Setting: Sri Devaraj Urs Medical College, Kolar, Karnataka, India.

Design: Retrospective study.

Materials and Methods: Medical records of cataract and aphakic patients who underwent posterior fixation of iris claw lens over a five-year period were reviewed for collection and analysis of data.

Results: The study comprised 138 eyes of $57.25 \%$ female and $42.75 \%$ male with mean age of $62.84 \pm 8.649$ years. The follow up period was 6 to 24 months. The mean preoperative CDVA was $0.44 \pm 0.23 \operatorname{logMAR}$. After 1 year of follow up period the mean postoperative CDVA was $0.3 \pm 0.15 \log$ MAR which was statistically significant $(\mathrm{p}<0.05)$ when compared to the preoperative values. The final log MAR CDVA was good in $54.35 \%$, better in $41.3 \%$ and worse in $4.35 \%$ eyes. In the study mean preoperative Spherical Equivalent (SE) was $-2.22 \pm 3.28 \mathrm{D}$ and post-operative SE was $-1.02 \pm 2.78 \mathrm{D}$. There was significant decrease in SE postoperatively $(\mathrm{P}=0.01)$.

Conclusions: This study showed the merits of retrofixated iris claw lens over AC IOL and SF IOL in terms of simple technique, short learning curve, satisfying overall visual outcome, less risk of complications and good safety profile.
\end{abstract}

Keywords: Aphakia, Enclavation, Iris claw, Retrofixation.

\section{Introduction}

Visual rehabilitation in aphakic patients include spectacles or contact lenses. But implanting an intraocular lens (IOL) have many advantages over the former in eliminating perceptual problems and reducing disparity in image size. Complications with contact lens includes poor maintenance, giant papillary conjunctivitis, corneal abrasions and hypoxia.

In the absence of capsular bag, optical correction can be achieved by implanting an anterior chamber intraocular lens (AC IOL), fixing lenses to sclera with 10-0 prolene suture or fibrin glue (Scleral fixated intraocular lens -SF IOL) and /or anterior or retro fixated iris claw intraocular lens (RFIC IOL). Although these lenses were used successfully, but their long term safety is major concern. Angle fixated AC IOLs resulted in corneal endothelial decompensation, uveitis, secondary glaucoma, hyphema (UGH syndrome), iris capture or pupil decentration, cystoid macular edema (CME). Similarly, SF IOL implantation resulted in lens tilt, decentration, choroidal haemorrhage, CME, damage to ciliary body, retinal detachment along with suture related complications. ${ }^{1-3}$

RFIC IOLs have the advantage of posterior chamber location closest to the nodal point of the eye, with a lowrisk, prevents tilting by enhanced stability and reduces the glare phenomenon, which is characteristic of the lens being implanted in the anterior chamber. ${ }^{4-6}$

Hence this retrospective study was conducted to study the indications, visual improvement, intraoperative and postoperative complications after posterior/retrofixated iris claw intraocular lens implantation in aphakic patients.

\section{Materials and Methods}

This retrospective case series study was approved by Institutional Ethics Committee. A total of 138 patients with uneventful retrofixation of iris claw lens over a five year period from January 2013 to January 2018 at R. L. Jallapa Hospital and Research Centre. Hospital medical records were reviewed for data collection and analysis.

Surgical Technique: After obtaining written informed consent about the risks and benefits of the surgery all patients were operated using the standard cataract surgical protocol under peribulbar anaesthesia by a single surgeon. A 6.0 to $6.5 \mathrm{~mm}$ sclerocorneal tunnel incision was made at 12 o'clock and 2 paracentesis at 3 o'clock and 9 o'clock position. Anterior chamber was reformed with Viscoelastic device (2\% Hydroxy propylmethyle cellulose -HPMC). A thorough anterior vitrectomy was done and pupillary miosis achieved with intracameral pilocarpine. Iris claw intraocular lens (Excel iris claw lens PIC 5590 model; Excel optics [p] Ltd., Chennai, India) was introduced into the anterior chamber with lens holding forceps.

Anterior chamber was deepened with HPMC and the haptics were enclaved posteriorly at 3 and 9 o'clock position by gently pushing the anterior surface of the iris with a Sinsky hook.

Anterior chamber was reformation after aspirating OVD and incision sutured with 2-3 interrupted 10-0 nylon sutures. Subconjunctival injection of dexamethasone and gentamicin was administered.

Postoperatively patients were instructed to instill Oflox D eye drops [Ofloxacin $0.3 \%+$ Dexamethasone $0.1 \%$, Cipla Ltd, Bengaluru, Karnataka] for 6 weeks in a tapering dose and followed up regularly for CDVA with 
Snellens chart and log MAR, refraction, Slit lamp examination for anterior and posterior segment findings and intraocular pressure measurement by non-contact tonometry.

Statistical Method Used: Data was entered into Microsoft excel data sheet and analyzed using SPSS 22 version software (IBM SPSS Statistics, Somers NY, USA). Paired t test is the test of significance and $P$ value of $\leq 0.05$ was considered as statistically significant.

\section{Results}

The study included 138 aphakic eyes of which $57.25 \%$ are female and $42.75 \%$ are male with mean age $62.84 \pm 8.649$ (SD) years. The follow up period was 6 to 24 months.

Indications: Table 1 shows the various indications for posterior iris claw lens implantation in patients. The main primary indication of this procedure was observed in Pseudoexfoliation syndrome with small pupil and zonular instability/ dialysis $(26.8 \%)$, posterior capsule rupture $(17.5 \%)$ followed by traumatic subluxated cataract $(13.8 \%)$ and as secondary indication in aphakia
(13\%) after previous cataract surgery.

Visual Outcome: Table 2 compares the preoperative and postoperative CDVA. After 1 year of follow up period the mean preoperative CDVA of $0.44 \pm 0.23$ $\log$ MAR and mean postoperative CDVA was $0.3 \pm 0.15$ $\log$ MAR. This improvement was statistically significant ( $\mathrm{p}<0.05$, paired $\mathrm{t}$ test) when compared to the preoperative values. The final log MAR CDVA was good in $54.35 \%$, better in $41.3 \%$ and worse in $4.35 \%$ eyes.

After preoperative and postoperative refraction, the mean Spherical Equivalent (SE) was $-2.22 \pm 3.28 \mathrm{D}$ and $-1.02 \pm 2.78 \mathrm{D}$ respectively. There was significant decrease in SE postoperatively $(\mathrm{P}<0.01)$

Complications: Fig. 1 shows postoperative complications the most common being oval pupil in 33 eyes $(23.9 \%)$, Iritis $(10.9 \%)$, Striae keratopathy $(8.7 \%)$, raised intraocular pressure $(7.9 \%)$ in the immediate postoperative period and Cystoid macular edema CME $(7.9 \%)$ at 6 months follow up. Six patients were reoperated for haptic disenclavation and IOL tilt.

Table 1: Indications for iris claw lens implantation

\begin{tabular}{|l|c|c|c|}
\hline \multicolumn{2}{|c|}{ Indications } & No: of eyes & \% \\
\hline \multirow{4}{*}{$\begin{array}{l}\text { Primary implantation } \\
\text { due to intraoperative complications }\end{array}$} & PXF* syndrome $^{*}$ & 27 & 26.8 \\
\cline { 2 - 4 } & Posterior Capsule Rupture & 24 & 17.5 \\
\cline { 2 - 4 } & Traumatic subluxated cataract & 19 & 13.8 \\
\cline { 2 - 4 } & Subluxated cataract due to PXF & 13 & 9.4 \\
\cline { 2 - 4 } & Zonular dehiscence & 10 & 7.2 \\
\cline { 2 - 4 } & Secondary glaucoma due to AC IOL & 2 & 1.4 \\
\cline { 2 - 4 } & Ectopia lentis (homocystinuria) & 1 & 0.7 \\
\hline Secondary implantation & Aphakia & 18 & 13 \\
\cline { 2 - 4 } & Traumatic subluxation of IOL & 8 & 5.8 \\
\cline { 2 - 4 } & Subluxated IOL due to PXF & 6 & 4.4 \\
\hline & Total & 138 & 100 \\
\hline
\end{tabular}

Table 2: Postoperative corrected distant visual acuity

\begin{tabular}{|l|c|c|c|c|}
\hline \multirow{2}{*}{ CDVA (log MAR) } & \multicolumn{2}{|c|}{ Preoperative } & \multicolumn{2}{c|}{ Postoperative } \\
\cline { 2 - 5 } & $\mathrm{N}$ & $\%$ & $\mathrm{~N}$ & $\%$ \\
\hline $0.17-0$ & 58 & 42.03 & 75 & 54.35 \\
\hline $0.5-0.3$ & 33 & 23.91 & 57 & 41.30 \\
\hline $1.0-0.6$ & 45 & 32.61 & 4 & 2.90 \\
\hline$>1.0$ & 2 & 1.45 & 2 & 1.45 \\
\hline Total & 138 & 100 & 138 & 100 \\
\hline
\end{tabular}




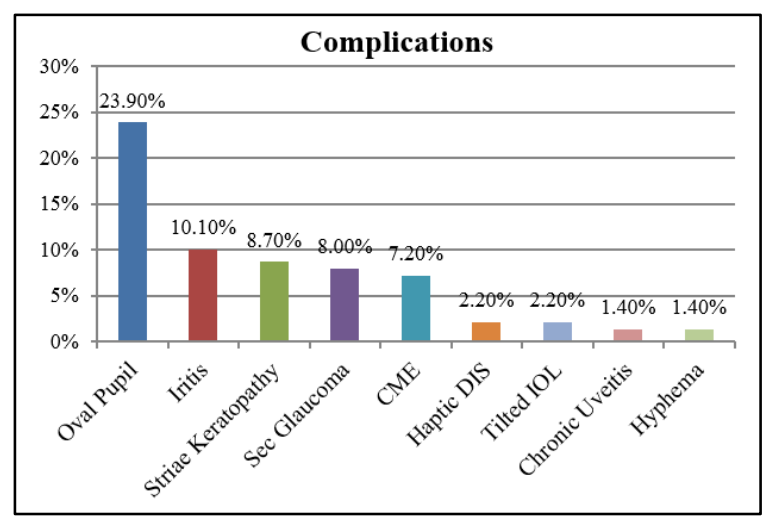

Fig. 1: Bar diagram showing complications among subjects

\section{Discussion}

This technique which was first reported by Andreas Mohr MD in 2002, ${ }^{7}$ offers several advantages and has been investigated further by several colleagues and is now frequently used. ${ }^{6,8,9}$

The best way of visual rehabilitation for an aphakic patient is with retropupillary iris claw fixation, its safety and efficacy is studied by several authors and found it to be safe procedure. ${ }^{10-13}$

During enclavation the haptics of the iris claw lens is tucked to a fold of mid peripheral iris stroma, which is less mobile, vascularized and less reactive. ${ }^{14,15}$ Enclaving to the posterior surface of the iris allows us to achieve a safe and stable fixation.

Main indication of this procedure was observed in Pseudoexfoliation syndrome with zonular instability/ dialysis $(26.8 \%)$ and posterior capsule rupture $(17.5 \%)$ followed by traumatic subluxated cataract $(13.8 \%)$ and aphakia (13\%) similar to other studies. ${ }^{16,17}$ One case of homocystinuria with inferior dislocation of clear lens was observed in a female who reported late with poor vision [Table 1]

Mean preoperative CDVA was $0.44 \pm 0.23$ LogMAR and mean postoperative CDVA at 1 year was $0.3 \pm 0.15$ Log MAR. Out of 138 eyes, 75 eyes $(54.35 \%)$ maintained preoperative visual acuity and 57 eyes $(41.3 \%)$ had improvement in visual acuity. Four cases had poor vision due to chronic uveitis and CME despite prompt treatment. [Table 2] comparable to a study conducted by Gonnermann et al. ${ }^{18}$ Another study showed comparable result, were the mean preoperative CDVA was $0.30 \pm 0.48 \log$ MAR and postoperative acuity at 1 year was $0.27 \pm 0.46 \operatorname{LogMAR}(P=0.07) .{ }^{17}$

In the study mean Preoperative Spherical Equivalent (SE) was $-2.22 \pm 3.28 \mathrm{D}$ and post-operative SE was -1.02 \pm 2.78 D. There was significant decrease in SE postoperatively

$(\mathrm{P}=0.01)$. Similar studies showed a mean preoperative astigmatism of $1.43 \pm 1.94 \mathrm{D}$ and postoperative value of $1.85 \pm 2.16 \mathrm{D}(P<0.05) .{ }^{13,16}$

The most common immediate postoperative complications observed in our study was oval pupil in 33 eyes (23.9\%), Iritis (10.9\%), Striae keratopathy (8.7\%), Hyphema $2(1.4 \%)$ Secondary glaucoma $(7.9 \%)$ which subsided with prompt topical antibiotic steroid and antiglaucoma medications in a week. [Table 3]

Ovalization of iris was also the commonly observed complication with no influence on the postoperative vision in other studies. ${ }^{18-20}$

Studies by Rufer et $\mathrm{al}^{21}$ and Anbari \& Lake ${ }^{22}$ showed similar results were secondary glaucoma was noticed at 1 week follow up that responded well with topical medications unlike chronic secondary glaucoma reported by Schallenberg et al. ${ }^{20}$

Delayed complication noticed in our study which resulted in poor post-operative vision was CME observed in 11 eyes (7.9\%), out of which 9 eyes recovered by 3-4 months and chronic uveitis in 2 eyes. [Fig. 1]

The incidence of disenclavation and IOL tilt observed at 2 months' visit was less $4.2 \%$ (6 eyes) when compared to Ramon et $\mathrm{al}^{23}$ and Sri Ganesh et al, $15.6 \%$ and $8.3 \%$ respectively was attributed to postoperative trauma and iris atrophy and such patients were reoperated successfully with adequate iris tissue tuck to prevent further complications.

\section{Conclusion}

This study showed the merits of retrofixated iris claw lens over AC IOL and SF IOL in terms of satisfying overall visual outcome, less risk of complications and good safety profile.

\section{Financial Support and Sponsorship: Nil.}

\section{Conflict of Interest: Nil}

\section{References}

1. Dick HB, Augustin AJ. Lens implant selection with absence of capsular support. Curr Opin Ophthalmol. 2001;12(1):47-57.

2. Wagoner MD, Cox TA, Ariyasu RG, Jacobs DS, Karp CL. Intraocular lens implantation in the absence of capsular support: a report by the American Academy of Ophthalmology. Ophthalmology. 2003;110(4):840-59.

3. Dadeya S, Kamlesh K, Kumari SP. Secondary intraocular lens (IOL) implantation: anterior chamber versus scleral fixation long-term comparative evaluation. Eur $J$ Ophthalmol. 2003;13(7):627-33.

4. S. Mennel. Spektrum der Augenheilkunde. 2004;18:279283.

5. M.C. Gaillard \& T.J. Wolfensberger. Klin. Monatsbl. Augenheilkd. 2004;221(5):435-7.

6. T.H. Neuhann. Session 3-Q: Cataract: Cataract and IOL Technology, Best Paper of Session (BPOS) Winners, ASCRS Washington, 2005.

7. Mohr A, Hengerer F, Eckardt C. retropupillary fixation of the iris claw lens in aphakia; 1-year outcome of a new implantation technique. Ophthalmologe. 2002;99:580583.

8. S. Mennel. Spektrum der Augenheilkunde. 2004;18:279283.

9. M.C. Gaillard \& T.J. Wolfensberger. Klin. Monatsbl. Augenheilkd. 2004;221(5):435-7. 
10. Rao R, Sasidharan A. Iris claw intraocular lens: A viable option in monocular surgical aphakia. Indian $J$ Ophthalmol. 2013;61:74-5.

11. Patil KB, Meleth P, Shanker MP. Pars plana vitrectomy with posterior iris claw implantation for posteriorly dislocated nucleus and intraocular lens. Indian $J$ Ophthalmol. 2011;59:497-500.

12. Anbari A, Lake DB. Posteriorly enclavated iris claw intraocular lens for aphakia: Long-term corneal endothelial safety study. Eur J Ophthalmol. 2015;25:20813.

13. Gonnermann J, Klamann MK, Maier AK, Rjasanow J, Joussen AM, Bertelmann E, et al. Visual outcome and complications after posterior iris-claw aphakic intraocular lens implantation. J Cataract Refract Surg. 2012;38:2139-43.

14. Guell JL, Velasco F, Malecaze F, Vazquez M, Gris O, Manero F. Secondary Artisan-Verysiae aphakic lens implantation. J Cataract Refract Surg. 2005;31(12):226671.

15. Baykara M, Ozcetin H, Yilmaz S, Timucin OB. Posterior iris fixation of iris-claw intraocular lens implantation through a scleral tunnel incision. Am J Ophthalmol. 2007;144(4):586-91.

16. G Jayamadhury, Sudhakar Po i, K Vinaya Kumar, R Madhu Kumar, K C Divyansh Mishra, Srinivasa Rao Nambula. Retropupillary fixation of iris-claw lens in visual rehabilitation of aphakic eyes. Indian Journal of Ophthalmology. 2016;64:743-746.

17. Sri Ganesh, Sheetal Brar, Kirti Relekar. Long term clinical and visual outcome $\mathrm{f}$ Retrofixated Iris claw lenses implantation in complicated cases. The Open Ophthalmology Journal. 2016;10;111-118.

18. Gonnermann J, Klamann MK, Maier AK, Rjasanow J, Joussen AM, Bertelmann E, et al. Visual outcome and complications after posterior iris-claw aphakic intraocular lens implantation. J Cataract Refract Surg. 2012;38:2139-43.
19. M. Forlini, W. Soliman, A. Bratu, P. Rossini, G. M. Cavallini, and C. Forlini. Long term follow up of retropupillary iris-claw intraocular lens implantation: a retrospective analysis. BMC Ophthalmology. 2015;15:143.

20. Schallenberg M, Dekowski D, Hahn A, Laube T, Steuhl KP, Meller D. Aphakia correction with retropupillary fixated iris-claw lens (Artisan) - Long-term results. Clin Ophthalmol. 2013;8:137-141.

21. Rufer F, Saeger M, Nolle B, Roider J. Implantation of retropupillary iris claw lenses with and without combined penetrating keratoplasty. Grafes Arch Clin Exp Ophthalmol. 2009;247(4):457-62.

22. Anbari A, Lake DB. Posteriorly enclavated iris claw intraocular lens for aphakia: Long-term corneal endothelial safety study. Eur J Ophthalmol. 2015;25:20813 .

23. Anglada R, Castellvi J, Parera A, Sabala A. Inverted implantation of posterior iris fixated intraocular lens with $23 \mathrm{G}$ transconjunctival vitrectomy in the management of secondary implant -Technique and stability, astigmatism and endothelial loss outcomes. J Emmetropia. 2014;5(3):133-43.

How to cite this article: Sangeetha, Kanthamani, Laxman B. H, Meghana C. R. G. Outcomes after posterior fixation of iris-claw lens in aphakic eyes. Indian J Clin Exp Ophthalmol. 2018;4(4):499-502. 